\title{
WALLDYN simulations of global impurity migration in JET and extrapolations to ITER
}

\author{
K. Schmid ${ }^{1}$, K. Krieger ${ }^{1}$, S. W. Lisgo ${ }^{2}$, G. Meisl ${ }^{1}$, S. Brezinsek ${ }^{3,4}$ and JET Contributors *,4 \\ ${ }^{1}$ Max-Planck-Institut für Plasmaphysik, Boltzmannstraße 2, D-85748 Garching, Germany \\ ${ }^{2}$ ITER Organization, Route de Vinon sur Verdon, 13115 Saint Paul-lez-Durance, France \\ ${ }^{3}$ Institute of Energy and Climate Research - Plasma Physics, Forschungszentrum Jülich, 52425 \\ Jülich, Germany \\ ${ }^{4}$ EUROfusion Consortium, JET, Culham Science Centre, Abingdon, OX14 3DB, UK
}

Corresponding Author: Klaus.Schmid@ipp.mpg.de

\begin{abstract}
:
The migration of first wall material due to erosion, plasma transport and re-deposition is one of the key challenges in current and future fusion devices. To predict erosion/re-deposition patterns and to understand the underlying principal processes, the global simulation code WallDYN was developed. It couples the evolution of the first wall surface composition to plasma impurity transport. To benchmark the WallDYN model, it was applied to the JET ITER-Like Wall experiment (JET-ILW), which mimics the ITER first wall material configuration and is thus an ideal environment to validate the predictive significance of WallDYN calculations for ITER application. The WallDYN calculations show good agreement with the Be deposition patterns determined from JET-ILW post-campaign wall tile analysis. The WallDYN results on $\mathrm{W}$ erosion \& deposition also qualitatively match the post mortem patterns but quantitatively fall short by factor of 20 which can be consolidated by including the influence of ELMs on the W erosion source. Applying the same model and process physics as for the JET calculations, the impurity migration and resulting fuel species co-deposition in ITER for different wall configurations and background plasmas was calculated. The simulations show that $\mathrm{C}$ containing wall configurations lead to unacceptable $\mathrm{T}$ retention whereas for the current ITER material choice (Be wall \& W divertor) co-deposition will not limit the ITER operation. However the erosion of $\mathrm{W}$ by self-sputtering even without the contribution of ELMs could hamper ITER operation under certain plasma scenarios.
\end{abstract}

${ }^{*}$ See the Appendix of F. Romanelli et al., Proceedings of the 25th IAEA Fusion Energy Conference 2014, Saint Petersburg, Russia 


\section{Introduction}

The migration of first wall material due to erosion, plasma transport and deposition is one of the key challenges in current and future fusion devices. It affects the lifetime of wall components in net erosion regions and can lead to formation of mixed material layers and strong fuel retention via co-deposition in net deposition regions of the first wall. To predict erosion/(re)deposition patterns and to understand the underlying principal processes, a global simulation code is required that couples the evolution of the first wall surface composition to plasma impurity transport. To that end, the WallDYN [1] code was developed. It maintains a strict global material balance of all eroded and (re-)deposited material and allows the tracking of the chain of subsequent erosion/(re-)deposition/re-erosion steps that define where material is finally netdeposited or net-eroded. WallDYN couples state of the art models for the surface processes (e.g. erosion, reflection, implantation, sublimation) with material redistribution data from trace impurity plasma transport models in a fully self-consistent simulation. For each plasma exposed location on the discretized first wall contour it calculates the time evolution of both the composition of elements in the surface and in the incident particle flux. Thus intrinsically including impurity erosion and self-sputtering. The fuel retention rate via co-deposition can be derived from the calculated layer growth rate by multiplying with the hydrogen concentration in the layer, which can be taken from scaling laws fitted to lab experiments [3]. To benchmark the WallDYN model, it was applied to the JET ITER-Like Wall experiment (JET-ILW), which mimics the ITER first wall material configuration and is thus an ideal environment to validate the predictive significance of the WallDYN deposition/retention calculations for ITER application. In [2] the light impurity migration (Be, C) and resulting (co-)deposition was found to match the experimental data from post mortem analysis and gas-balance measurements rather well. In this paper the WallDYN calculation results on $\mathrm{W}$ erosion \& deposition for JET-ILW will be presented and compared to post mortem analysis results from [16]. Applying the same model and process physics as for the JET calculations, the Be deposition patterns and resulting fuel species co-deposition in ITER for different wall configurations and background plasmas was calculated. The background plasmas used in DIVIMP to calculate the redistribution information span a large range of plasma parameters at the wall.

In addition to (co-)deposition WallDYN also calculates the erosion rate for all wall elements. For ITER the erosion of $\mathrm{W}$ is a key parameter since excessive $\mathrm{W}$ sputtering could severely limit the operational space due to the tendency of $\mathrm{W}$ to accumulate and radiate in the plasma core. The paper will first present JET-ILW WallDYN benchmarking calculations on W migration, showing how the shortfall in the erosion in the calculations compared to experiment can be explained by the contribution of ELMs to the $\mathrm{W}$ erosion source. Then the predictions for ITER w.r.t Be deposition and fuel retention by co-deposition will be discussed. Finally some results 
on the erosion of $\mathrm{W}$ by self-sputtering will be presented.

\section{Model Description}

The WallDYN model is described in detail in $[1,2]$ therefore only a short summary of the main concepts is given here.

WallDYN discretizes the wall into $\mathrm{N}$-wall elements and calculates the time evolution of the surface composition and the incident particle spectrum on each wall element due erosion, deposition and global particle transport.

The surface composition is described by the areal densities $\delta_{e i, w k}\left(m^{-2}\right)$ of the species $e i$ in on each wall element $w k$. The surface composition evolution is determined from a differential equation describing the flux balance of material loss (erosion \& sublimation $\Gamma_{e i, w k}^{E R O}$ ) and gain (deposition $\Gamma_{e i, w k}^{D E P}$ ) due to the local incident particle spectrum. The rate determining parameters (erosion \& reflection yield, sublimation rate) for the surface composition evolution are calculated from scaling laws. These scaling laws are determined from fits to experimental data and output from state of the art models (e.g. SDTRIM.SP $[10,11])$.

The incident particle spectrum is given by the flux $\Gamma_{e j, w l}$ of element $e j$ on wall element $w l$ $\left(m^{-2} s^{-1}\right)$. The incident flux evolution is calculated from the fluxes from each wall element into the plasma due to reflection \& erosion multiplied by the material re-distribution matrix. This redistribution matrix is a parametrization of the output of the trace impurity migration code DIVIMP [6]. It describes how much material that is emitted from wall element $w k$ into the plasma ends up on wall element $w l$. Since the flux of material into the plasma depends on the current surface composition $\delta_{e i, w k}(t)$ and incident particle spectrum $\Gamma_{e j, w l}(t)$ this matrix defines a linear equation system from which the equilibrium $\Gamma_{e j, w l}(t)$ can be calculated given the current $\delta_{e i, w k}(t)$. By combining the differential equation for the surface composition evolution with the linear equation of the flux evolution a differential algebraic equation (DAE) system can be derived that describes the coupled evolution of surface and plasma.

The fact that both the surface processes and the plasma transport processes are described by parameterizations of the calculation results from sophisticated codes allows to couple surface processes and plasma transport without the use of an iterative scheme. This avoids the problems associated with discretizing time or sampling distributions which make iterative schemes prone to artifacts.

The most important input for the impurity transport calculation by DIVIMP is the background plasma. It also defines the constant background plasma flux (D-ions, D-CX) and plasma parameters $(\mathrm{Te}, \mathrm{Ti})$ at the wall. For the JET calculations the background plasma was calculated using a quasi 1D "onion-skin" plasma model (OSM) [12]. While the onion-skin model only solves a simplified version of the Braginskii equations, it allows a very close match to the ex- 
perimentally observed plasma parameters because measurements of temperature and density at the divertor plates and the outer mid-plane are used as boundary conditions.

For the ITER calculations the approach is slightly different: The near SOL plasma is taken from a SOLPS [13] calculation, while the far SOL plasma is based on a simple isothermal, purely convective plasma model that prescribes plasma parameters that are consistent with observed experimental trends [9]. See also [2] for further details on the different ITER background plasmas cases.

For both JET and ITER all the background plasmas are defined on a computational grid that tightly matches the entire first wall without gaps. This allows to directly extract wall fluxes around the entire poloidal circumference without the need the extrapolate plasma fluxes across gaps between the calculation grid and the wall. In the DIVIMP calculations the perpendicular transport of impurities is controlled by the perpendicular diffusion coefficient $D_{\text {Perp }}\left(m^{2} s^{-1}\right)$. In the calculations presented here $D_{\text {Perp }}=1.0 \mathrm{~m}^{2} \mathrm{~s}^{-1}$ was used.

\section{Results and Discussion}

The main questions that have been addressed in the WallDYN calculations for ITER are: The Be deposition in the divertor and resulting retention by co-deposition; The erosion and migration of $\mathrm{W}$. Therefore to judge the predictive significance of the WallDYN calculations it was compared to experimental data from the JET-ILW campaign. In [2] the successful benchmark of WallDYN against the measured Be migration and fuel retention by (co-)deposition was already presented. In the next subsection the WallDYN results on $\mathrm{W}$ erosion \& deposition in the JET-ILW divertor will be compared against post mortem data from [16].

\subsection{WallDYN modeling of $\mathrm{W}$ erosion \& deposition in JET-ILW}

Two background plasmas were used in the comparison (See[2] for more details):

- 80295 an ohmic shot

- 83559 an H-Mode shot based on inter ELM profiles of $T_{e}$ and $N_{e}$

In Fig 1 the W erosion flux due to different incident species is shown for the ohmic shot 80295 and the inter ELM H-mode background plasma for shot 83559. As expected the W erosion is entirely determined by impurities mainly Be and $\mathrm{W}$ self-sputtering. The main $\mathrm{W}$ source location is the outer strike point located on tile 5 with a total peak value of $1.4 \times 10^{19} \mathrm{~m}^{-2} \mathrm{~s}^{-1} \approx 23 \mathrm{~nm} / \mathrm{s}$ for H-mode and a factor of 10 lower for the ohmic case. These high erosion rates are partially compensated by a very high re-deposited fraction which at the divertor targets is close to $80 \%$ leaving only a peak net erosion in the H-mode case of $4 \times 10^{18} \mathrm{~m}^{-2} \mathrm{~s}^{-1} \approx 6 \mathrm{~nm} / \mathrm{s}$. This however 
leaves the question how such large amount of re-deposited $\mathrm{W}$ material evolves over time and whether this will lead to a degraded surface layer with detrimental properties w.r.t. erosion and fuel retention. Also this large number of re-depositing particles is the reason for the high selfsputtering yield, since the returning $\mathrm{W}$ is ionized and thus accelerated by the sheath. WallDYN thereby assumes that the returning $\mathrm{W}$ ions are accelerated by the full sheath potential drop i.e. are assumed to be ionized outside the sheath potentially even to charge states $>1$. In Fig. 2 the net deposition of $\mathrm{W}$ as calculated by WallDYN is compared to post mortem data from ion beam analysis (IBA) of marker samples from the JET-ILW divertor. The marker samples were located around the poloidal circumference of the divertor (tile 1 to tile 8 ) with the exception of tile 5 for which no experimental information so far is available on the $\mathrm{W}$ erosion \& deposition. The details of the JET tile numbering scheme can be found for instance in [4]. In Fig. 2 only the data from the tiles were a measurable $\mathrm{W}$ deposition (above the detection limit of the applied IBA method) was found are shown.

During the campaign a total of $\approx 13 \mathrm{~h}$ of divertor time were accumulated. The comparison in Fig. 2 shows a qualitative match between the WallDYN calculations and the post mortem data: Most W-deposition occurs on tile 6 next to the strike point on tile 5 and on the tile 1 "apron" region on the entrance to the inner divertor. The calculations predict the strongest deposition in the private flux region next to the strongest $\mathrm{W}$ erosion source, the strike point on tile 5 . However no post mortem data is available for tile 5 and thus no comparison of the erosion/deposition pattern on tile 5 can be made.

The quantitative comparison between the post mortem data and the WallDYN calculations is subject to large uncertainties: The post mortem data are the result of $\approx 13 \mathrm{~h}$ of divertor operation with different plasma conditions (power, fluxes, strike point location etc.). Still a rough comparison can be made by comparing the resulting $\mathrm{W}$ erosion \& deposition after an assumed $13 \mathrm{~h}$ of steady state plasma operation with the background plasma from shot 80295 or 83559. These two plasmas both have the strike points on tile 3 and 5 which is a configuration that was dominantly used during the campaign [5] thus they can be assumed to be a reasonable proxy for actual plasma operation. Using this assumption the absolute $\mathrm{W}$ deposition shown in Fig. 2 were derived. For the ohmic discharge the difference between the calculated deposition ranges from a factor 20 on tile 6 to a factor 100 on tile 3. For the inter ELM H-mode background plasma the discrepancy is in the order of a factor of 20 throughout the poloidal circumference of the divertor. This discrepancy is not unexpected: The low power ohmic discharge is hardly representative w.r.t to $\mathrm{W}$ erosion and the H-mode plasma lacks the influence of ELM's which probably dominate the $\mathrm{W}$ erosion in the experiment.

ELM's influence the erosion \& deposition in different ways: Most prominently they affect the source strength by increasing the physical sputtering due to the hot ions present during an ELM. But the ELM may also affect the transport of $\mathrm{W}$ away from its source but this is beyond current 
impurity transport codes which typically operate on steady state 2D background plasmas. Therefore to obtain a first order estimate if ELM's can account for the difference between the calculated absolute amounts of $\mathrm{W}$ deposition and the experimentally observed values, the influence of ELM's on the W source was calculated. Since WallDYN maintains a strict global material balance any increase in the source is directly reflected in the deposited amounts since eroded $\mathrm{W}$ is not lost from the machine. To estimate the increase in $\mathrm{W}$ erosion due to ELM to following assumptions were made: The ion impact energy during the ELM is comparable to the pedestal temperature of $\approx 1 \mathrm{keV}$ and the ion flux increases by a factor 5 compared to inter ELM conditions. Further the ELM's hit the target at a rate $\nu_{E L M}$ of $10 \mathrm{hz}$ and duration $\tau_{E L M}$ of $2 \mathrm{~ms}$. This change in the plasma parameters is of course only applied to a small ELM wetted area around the strike points of $2 \mathrm{~m}^{2}$ for JET-ILW and $13 \mathrm{~m}^{2}$ in ITER. Based on these assumption, which according to $[17,18]$ are reasonable values for JET-ILW ELMs, the W erosion source due ELM and inter ELM phase can be calculated from eq. 1

$$
\begin{aligned}
\delta^{E R O} & =n_{E L M}\left(\Gamma^{B A S E} Y^{B A S E}\left(\frac{1}{\nu_{E L M}}-\tau_{E L M}\right)+\Gamma^{E L M} Y^{E L M}\right) \\
n_{E L M} & =\text { number of elms } \\
\Gamma^{B A S E} Y^{B A S E} & =\text { inter ELM erosion flux } \\
\Gamma^{E L M} Y^{E L M} & =\text { ELM erosion flux }
\end{aligned}
$$

Defining $\alpha \equiv \frac{\Gamma^{E L M} Y^{E L M}}{\Gamma^{B A S E} Y^{B A S E}}$ and the ELM averaged erosion flux $\left\langle\Gamma^{E R O}\right\rangle=\frac{\delta^{E R O}}{\frac{n_{E L M}}{\nu_{E L M}}}$ the increase $R_{E R O}^{W}$ in the erosion flux relative to the inter ELM erosion flux can be written as in eq. 2.

$$
\begin{aligned}
R_{E R O}^{W} & =\frac{\left\langle\Gamma^{E R O}\right\rangle}{\Gamma^{B A S E} Y^{B A S E}} \\
& =\left(1+(\alpha-1) \nu_{E L M} \tau_{E L M}\right)
\end{aligned}
$$

To obtain an estimate for $\alpha$, the inter ELM erosion flux $\Gamma^{B A S E} Y^{B A S E}$ due to impurities was compared to the D only erosion flux $\left(=\Gamma^{E L M} Y^{E L M}\right)$ during the ELM. During the ELM, due to the orders of magnitude higher fluxes, D-ion sputtering dominates over impurity sputtering which currently can not reasonably be predicted. Therefore this estimate of $\alpha$ remains a lower boundary. In table I eq. 2 is used to estimate the increase of Be and W erosion due to ELM activity in JET-ILW H-Mode (83559) and ITER. For ITER an average value over the different available (see above) ITER cases is given, excluding the cases with a separatrix distance of 4 $\mathrm{cm}$ for which excess W self sputtering occurred (see below). 


\begin{tabular}{lll}
\hline Case & Be & W \\
\hline JET-ILW H-Mode & 3 & 17 \\
\hline ITER & 1 & 5
\end{tabular}

TABLE I: Estimates OF THE RATIO OF THE EROSION FLUX DURING- AND INTER ELM FOR BE AND W FOR JET-ILW AND ITER CONDITIONS

The numbers in table I of course depend on the afore mentioned underlying assumptions in particular on the choice of the D ion energy during the ELM but they show a fundamental trend: ELM erosion is most important for predicting $\mathrm{W}$ erosion in current machines like JET. In ITER or for Be it is of little importance since the inter ELM erosion is already quite high. For JET-ILW H-mode case the estimated increase in the W source due to ELMs and therefore also in the deposited amount is a factor 17. This is close to discrepancy (factor 20) found in the comparison between WallDYN calculations and the post mortem results. Thus this difference can be attributed to ELM erosion. The fact that the migration patters of $\mathrm{W}$ are well reproduced by the trace impurity transport calculations in WallDYN, suggest that ELMs do not dominate the transport but mainly increase the $\mathrm{W}$ impurity source.

\subsection{Predictions for ITER}

To calculate the Be deposition patterns in ITER for the different background plasmas the WallDYN surface model was adopted to also include Be sublimation as an additional loss channel. The steady state surface temperatures at the divertor targets reaches temperatures of up to $\approx 1400 \mathrm{~K}$ where Be sublimation is the dominant loss channel. The main wall was assumed to remain at $385 \mathrm{~K}$ where sublimation is negligible. WallDYN calculations for different first wall material configurations were performed: A full Be first wall and $\mathrm{W}$ divertor with CFC strike point areas $=$ CASE-A, a full Be first wall and a full $\mathrm{W}$ divertor $=\mathrm{CASE}-\mathrm{B}$ and full $\mathrm{C}$ first wall and divertor $=$ CASE-C.

In Fig. 3 the calculated Be deposition along the ITER poloidal circumference is shown for the ITER reference wall configuration CASE-B (no C). The X-axis is the WallDYN wall element index and along the $\mathrm{Y}$-axis the different background plasma cases are varied. The Be deposition is plotted in units of Be atoms per meter toroidal length (m-tor) and seconds. This value is obtained from the calculated Be deposition flux (Be $/ \mathrm{m}^{2} \mathrm{~s}$ ) by multiplying it with the poloidal length of the wall element. The calculation predicts significant Be deposition on upper dump plate and divertor baffles. The reason for the strong deposition at the upper dump plate is the secondary upper $\mathrm{X}$ point which in ITER is located close to the upper dump plate, thus essentially forming two additional strike points on the upper dump with high local fluxes. While this basic deposition pattern is found for all background plasmas, there still exists a 
strong variation in the absolute Be deposition for different background plasmas. For instance along the inner and outer targets the Be deposition locally varies by almost a factor 10 from the average $\left(\approx 0.5 \times 10^{19} m-t^{-1} s^{-1}\right)$ to the maximum $\left(\approx 3 \times 10^{19} m-t^{-1} s^{-1}\right)$ deposition rate when averaging over all background plasma cases. This variation in the Be deposition is also found in the fuel retention rate as discussed below.

The fuel retention due to co-deposition in ITER for the different first wall configurations (CASEA, CASE-B and CASE-C) is shown in Fig. 4. There the experimental (from [8]) and calculated JET-ILW and JET-C values together with the extrapolation to ITER are compared. The simulations showed that CASE-B has the lowest retention due to co-deposition whereas CASE-A and CASE-C feature on average a 10 and 100 times higher retention rate respectively. For case C only 100 to 700 full 400 second ITER discharges would be possible before hitting the T-limit of 700g (T:D ratio = 50:50) whereas for CASE-B between 3000 and 20000 full 400 second ITER discharges are possible depending on the plasma configuration. These numbers do not include the influence of cleaning discharges. It should also be noted that the difference between CASE-A and CASE-C is quite small despite the fact that there is much more $\mathrm{C}$ in CASE-C. As the dominant erosion occurs at the $\mathrm{C}$ strike points the additional contribution to co-deposition by $\mathrm{C}$ eroded from the main wall is small.

These numbers are in line with previous estimates in [14] based on a simple first wall flux scaling. However independent of the wall material configuration, the different background plasmas result in a factor $\approx 10$ variation in co-deposition despite similar total wall fluxes. These strong variations show that a simple wall flux scaling is not enough for predicting retention in ITER for various plasma conditions. Still, for the current ITER material choice (CASE-B) fuel retention by co-deposition will not limit the ITER operation.

During the ITER calculations for the different background plasma cases, excessive W self sputtering was found for certain background plasmas. Scenarios with high edge temperatures/densities result in sputtered $\mathrm{W}$ returning to the wall at high charge states $(>8+)$ where they, due to sheath acceleration, result in $\mathrm{W}$ self-sputter yields close to unity. This problem is aggravated by the expected oblique ion impact angles. In the calculation oblique impact angles of $40^{\circ}$ were chosen based on results and modeling in [15]. At first the reason for this remained unclear since the local plasma parameters according to "conventional" self-sputtering estimates should not lead to excessive self-sputtering. In these "conventional" self-sputtering estimates the eroded flux $\Gamma^{E r o}$ of an element due to the background plasma flux $\Gamma^{\text {Back }}$ and the self-sputtering due to a (prompt) re-depositing flux $\Gamma^{\text {Self }}$ is calculated as in Eq. 3 


$$
\begin{aligned}
\Gamma^{E R O} & =\Gamma^{\text {Back }} Y^{\text {Back }}+\xi \Gamma^{E R O} Y^{\text {Self }} \\
\Gamma^{E R O} & =\frac{\Gamma^{\text {Back }} Y^{\text {Back }}}{1-\xi Y^{\text {Self }}} \\
Y^{\text {Back }} & =\text { Background flux sputtering yield } \\
Y^{\text {Self }} & =\text { re-depositing flux self-sputtering yield } \\
\xi & =\text { re-depositing fraction }
\end{aligned}
$$

In Eq. 3 the erosion flux diverges when $Y^{\text {Self }} \rightarrow \frac{1}{\xi}$ which is commonly referred to as run-away self sputtering. For run-away self sputtering to occur according to Eq. $3 Y^{\text {Self }} \gg 1$ is required since $\xi \ll 1$. Since the self-sputtering species is usually assumed to return immediately by prompt re-deposition at a charge state of +1 , a very high $T_{e}$ is required to reach $Y^{\text {Self }} \gg 1$. This makes run-away self sputtering hard to reach in these "conventional" self-sputtering estimates. In WallDYN the incident fluxes $\Gamma_{e i, q i, w r}^{\mathrm{In}}$ of element ei at charge sate qi onto wall element wr are calculated according to Eq. 4. 
$\Gamma_{e i, w s}^{\text {Ero }}=$ Erosion flux of component ei on wall element ws

$$
=\sum_{e j=0}^{\mathrm{NElem}} C_{e i, w s}\left(\sum_{q j=0}^{\mathrm{NChrg}_{\mathrm{ej}}} \Gamma_{e j, q j, w s}^{\mathrm{In}} * Y_{e i, e j}\left(E_{q j, w s}, \delta_{e k \ldots N}\right)\right)
$$

$$
\begin{aligned}
Y_{e i, e j}\left(E_{q j, w s}, \delta_{e k \ldots N}\right)= & \text { Sputter yield of element ei by element ej on wall element ws } \\
& \text { as function of energy E and composition } \delta_{e k} \text { (Areal density of element ek) } \\
C_{e i, w s}= & \text { Concentration of element ei on wall tile ws } \\
\Gamma_{e i, w s}^{\text {Refl }}= & \text { Reflected flux of component ei from wall element ws } \\
= & \sum_{q i=0}^{\text {Chrg }_{\text {ei }}} \Gamma_{e i, q i, w s}^{\mathrm{In}} * R_{e i, w s}\left(E_{q i, w s}, \delta_{e j \ldots N}\right) \\
R_{e i}\left(E_{q i, w r}, \delta_{e j \ldots N}\right)= & \text { Reflection yield of element ei on wall tile wr } \\
\Gamma_{e i, q i, w r}^{\mathrm{In}}= & \text { Linear equation for evolution of flux } \\
& \text { of element ei, charge state qi on wall element wr } \\
= & \sum_{w s=1}^{\text {NElem }}\left(\Gamma_{e i, w s}^{\text {Ero }}\left(\Gamma_{e j, q j, w s}^{\mathrm{In}}\right)+\Gamma_{e i, w s}^{\text {Refl }}\left(\Gamma_{e i, q i, w s}^{\mathrm{In}}\right)\right) * \xi_{e i, q i, w s, w r} \\
= & \text { Charge state resolve redistribution matrix } \\
= & \text { Fraction of element ei } \\
\xi_{e i, q i, w s, w r} & \text { emitted from ws ending up on wr at charge state qi }
\end{aligned}
$$

Eq. 4 describes the redistribution material emitted (due to erosion and reflection) from all wall elements ws onto all wall elements wr due to plasma impurity transport (see also [1]). With respect to self-sputtering this means that the influx onto a wall element is not only dependent on the amount of atoms eroded locally which come back to the same location by (prompt) re-deposition. It also depends on what is eroded on other parts of the first wall, travels through the SOL before it potentially self-sputters at a particular wall element. Thus this more refined approach used in WallDYN not only includes reflection but also the transport of the emitted particle through the plasma. This means that the charge state of the impinging, self-sputtering, particles can be larger than unity which has a huge impact on self sputtering since the sheath acceleration of highly charged ions can increase the sputtering yield significantly. Therefore the erosion by self sputtering not only depends on the local plasma parameters (i.e. $T_{e}$ ) at the wall location, but also on the SOL plasma in the vicinity. This fact explains why certain ITER scenarios with reduced distance between the primary and secondary separatrix are prone to excessive self sputtering: The close proximity of the hot plasma to the wall results in a 
significant increase in the wall flux of high W charge states.

This effect is not expected in current machines, and has not occurred in any other WallDYN calculation performed so far for JET or AUG. The reason is that such a high plasma density combined with a high plasma temperature so close to the wall is not possible in current machines. Therefore no benchmark exists, to test these WallDYN predictions against. None the less these results should not be ignored and be taken into consideration during ITER scenario development.

\section{Summary}

The global impurity transport code WallDYN was benchmarked against experiments in JETILW. The W erosion \& deposition calculations were compared to post mortem analysis of W deposition. WallDYN matched the qualitative deposition pattern well but a quantitative comparison falls short by a factor 20 which can be consolidated by including the contributions of ELMs to the W erosion source.

Applying the same process physics as for the JET-ILW calculations WallDYN was used to predict Be deposition patterns and the resulting co-deposition for ITER, for a wide range of ITER plasmas conditions. These calculations showed that $\mathrm{C}$ leads to unacceptably high retention rates whereas the current ITER material choice (Be main wall and full $\mathrm{W}$ divertor) will not limit ITER operation.

These WallDYN calculations have also shown that for certain plasma configuration excessive W self sputtering may occur even with the contribution of ELMs. Scenarios with a small distance between the primary and secondary separatrix result in eroded W returning to the wall at very high charge states resulting in excessive W self-sputtering. Thus such plasma scenarios may not be possible in ITER.

\section{Acknowledgments}

This work was supported by EURATOM and carried out within the framework of the European Fusion Development Agreement. This work has been carried out within the framework of the EUROfusion Consortium and has received funding from the European Union's Horizon 2020 research and innovation programme under grant agreement number 633053. The views and opinions expressed herein do not necessarily reflect those of the European Commission. The views and opinions expressed herein do not necessarily reflect those of the ITER Organization. 


\section{References}

[1] K. Schmid et al., Journal of Nuclear Materials 415 (2011) S284-S288

[2] K.Schmid el al., "PSI-2014: Quantitative modeling of fuel retention in the JET-C and JET-ILW wall configurations by WallDYN and predictions for ITER" Accepted in Journal of Nuclear Materials (2014)

[3] G. De Temmerman et al., Nucl. Fusion 48 (2008) 075008

[4] K. Heinola et al. "Fuel Retention in JET ITER-Like Wall from Post-Mortem Analysis" Submitted to Journal of Nuclear Materials (2014)

[5] J. P. Coad et al. Phys. Scr. T159, (2014) 014012

[6] P. C. Stangeby, J. D. Elder, J. Nucl. Mat. 196-198 (1992) 258

[7] P. C. Stangeby, ISBN 075030559 2, IOP Publishing Ltd 2000, Chap. 12

[8] S. Brezinsek et al., Nuclear Fusion 53 (2013) 083023

[9] S. Lisgo et al., J. Nucl. Mat. 438 (2013) 580

[10] W. Möller, W. Eckstein, J. P. Biersack, Comp. Phys. Commun. 51 No. 8, 1988, 355

[11] A. Mutzke, R. Schneider, W. Eckstein, MPI für Plasmaphysik IPP-Report 12/8, (2011)

[12] S. Lisgo, P.C. Stangeby, J.D. Elder et al., J. Nucl. Mat. 337 (2005) 256

[13] R. Schneider, X. Bonnin, et al., Contrib. Plasma Phys. 46 (2006) 191

[14] J. Roth et al., J. Nucl. Mater. 390-391, (2009), 1

[15] K. Schmid et al., Nucl. Fusion 50 (2010) 105004

[16] M. Mayer, S. Krat, W. Van Renterghem, A. Baron-Wiechec, S. Brezinsek, I. Bykov, P. Coad, Yu. Gasparyan, K. Heinola, J. Likonen, A. Pisarev, C. Ruset, G. de Saint-Aubin, A. Widdowson, and JET Contributors, Erosion and deposition in the JET divertor during the first ILW campaign, "To be published in the proceedings of PFMC 2015"

[17] B. Sieglin et al., Plasma Phys. Control. Fusion 55 (2013) 124039

[18] C. Silva et al., J. Nucl. Mat. 390 (2009) 355 

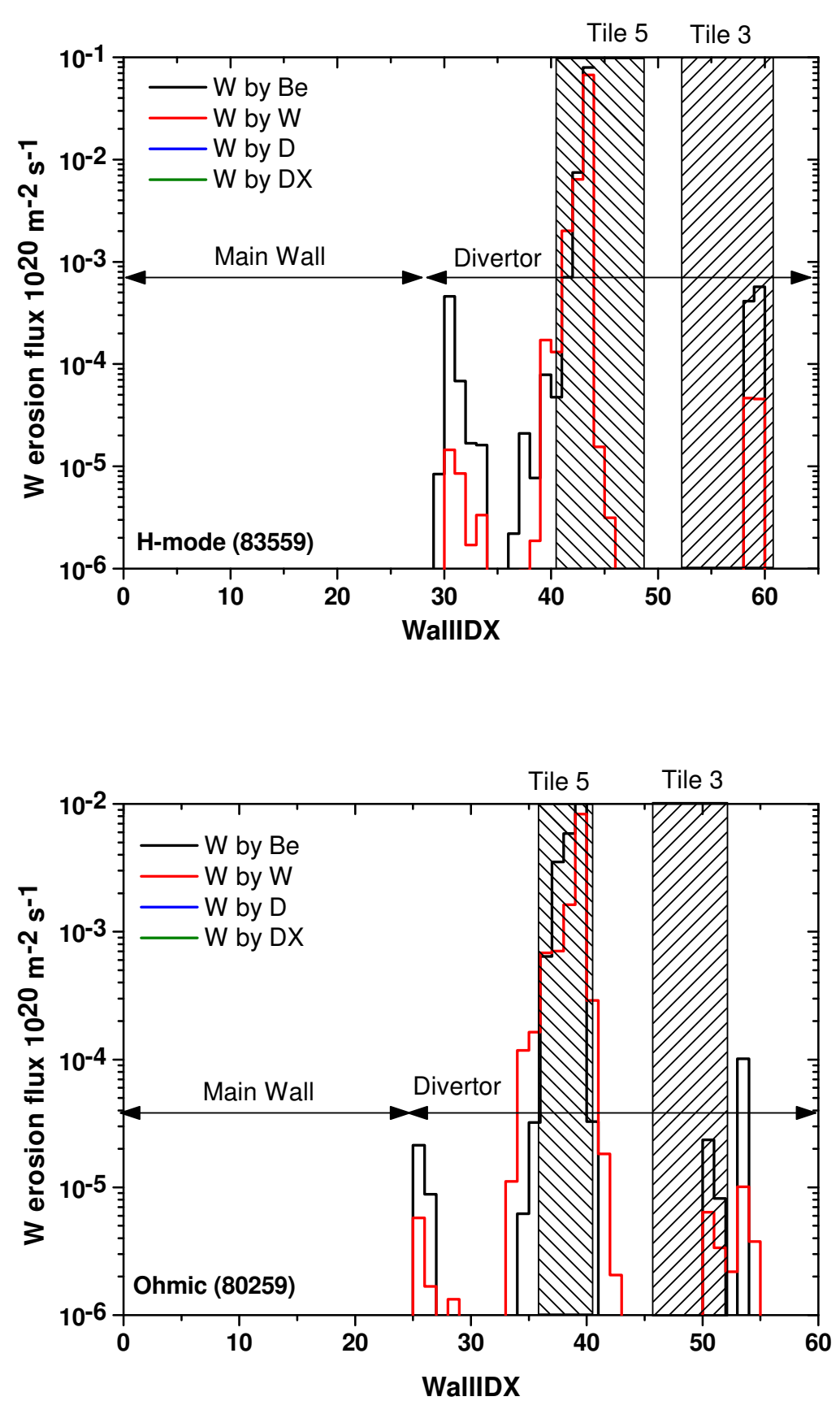

FIG. 1: Poloidal variation of $W$ erosion flux due to different impurity ions in JET-ILW configuration. Shown are results for H-Mode (83559) and Ohmic (80295) background plasmas. 

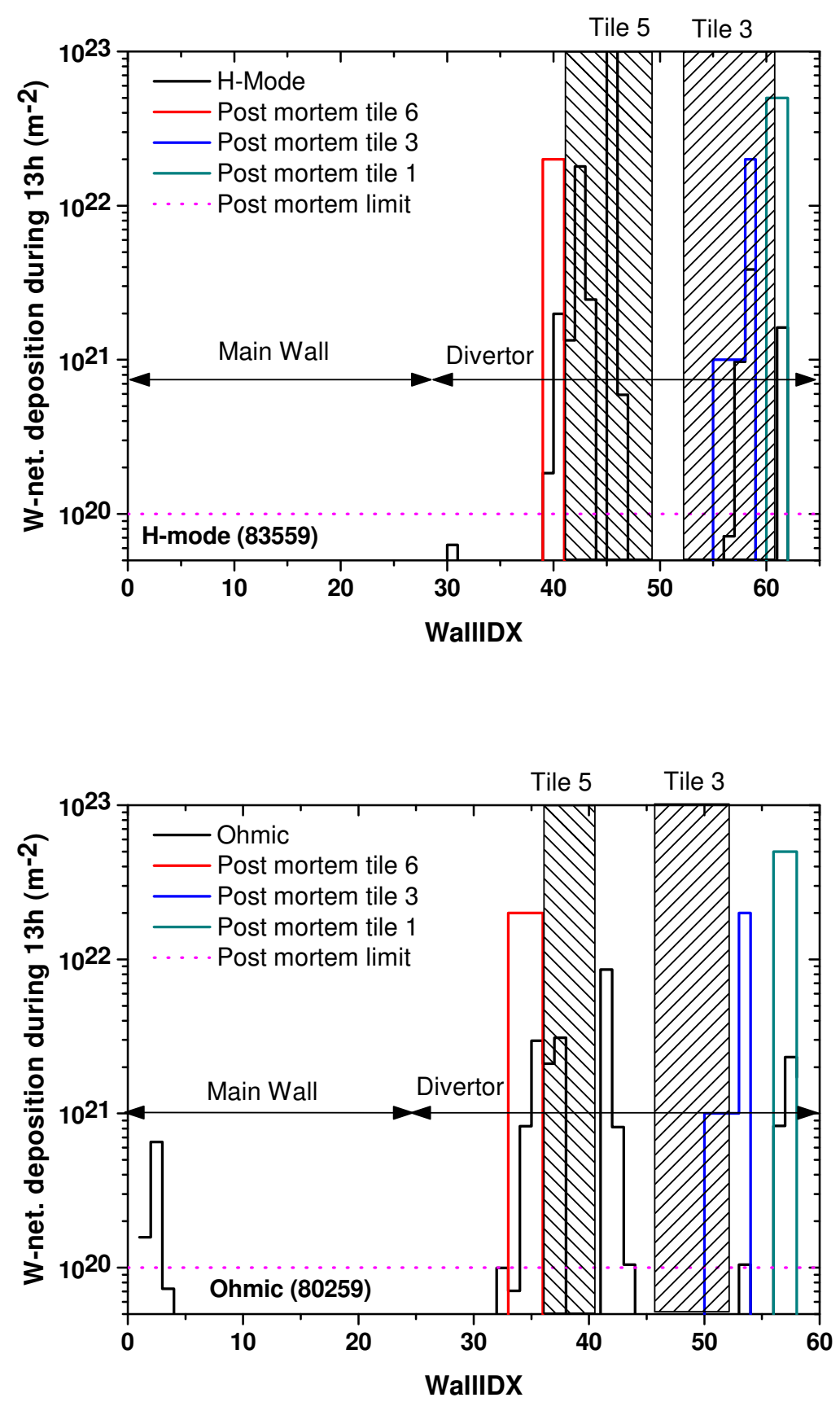

FIG. 2: Poloidal variation of $W$ deposition in JET-ILW configuration. Comparison of post mortem with WallDYN calculated data for H-Mode (83559) and Ohmic (80295) background plasmas 


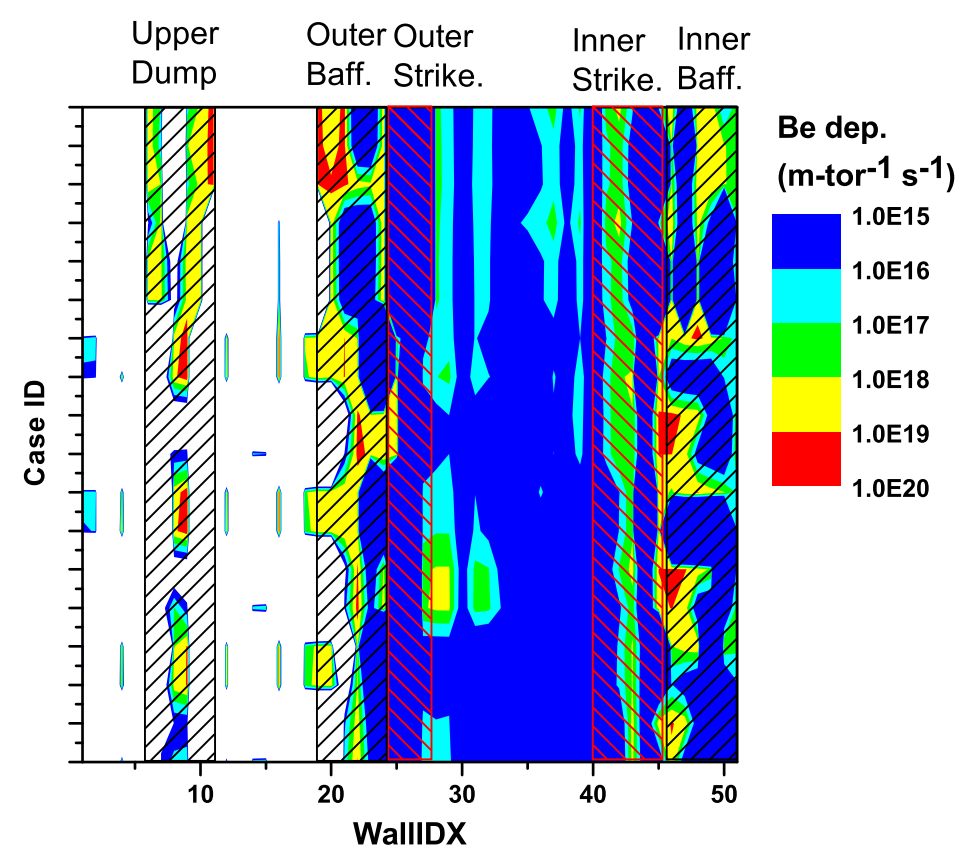

FIG. 3: Comparison of WallDYN calculated Be deposition along the ITER poloidal circumference. The different background plasmas varies along the Vertical axes.

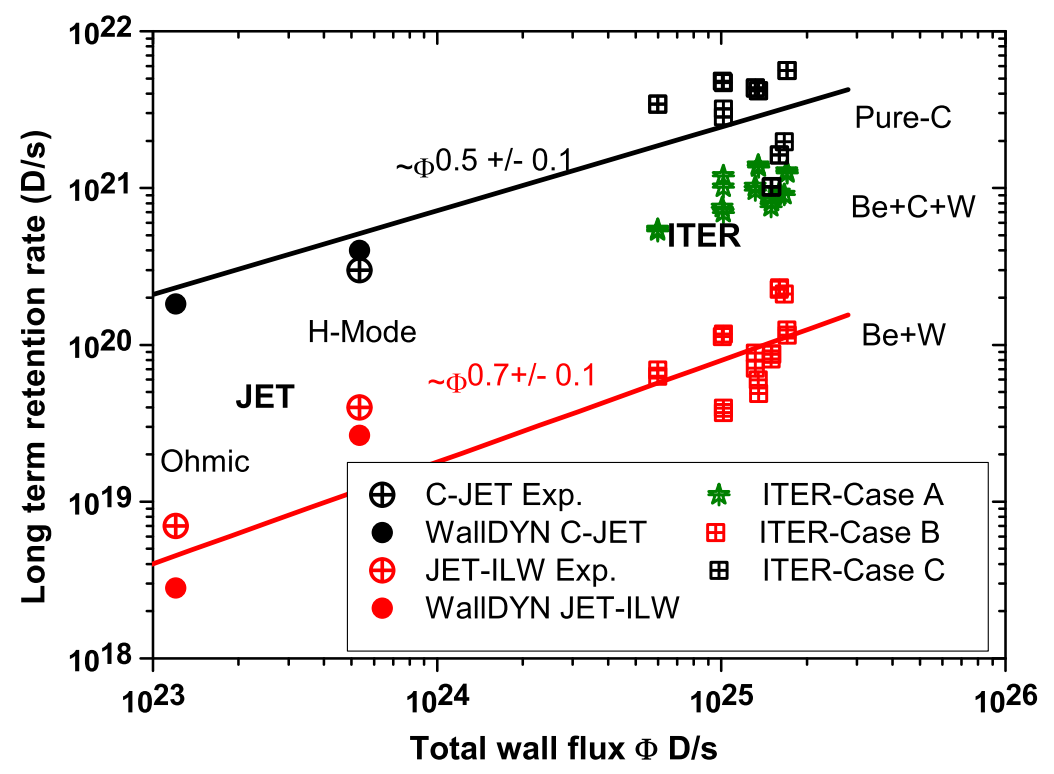

FIG. 4: Comparison of WallDYN calculated retention rates for ITER and JET. Black: Pure $C$, Green: $C+B e+W$, Red: $B e+W$ 